\title{
PREVALÊNCIA DE LESÃO POR PRESSÃO EM PACIENTES INTERNADOS EM HOSPITAL PRIVADO DO ESTADO DE MINAS GERAIS
}

Fernanda de Carvalho ${ }^{1}$

Miguir Terezinha Vieccelli Donoso

Braulio Roberto Gonçalves Marinho Couto Selme Silqueira de Matos ${ }^{1}$

Lilian Kelly Barbosa Lima ${ }^{4}$

Érica Pertussati ${ }^{1}$

\author{
https://orcid.org/0000-0003-3239-705X \\ http://orcid.org/0000-0002-5497-9520 \\ https://orcid.org/0000-0002-5314-5161 \\ http://orcid.org/0000-0002-4248-7107 \\ https://orcid.org/0000-0003-0858-1869 \\ http://orcid.org/0000-0002-6621-084X
}

Objetivos: Estimar a prevalência de lesão por pressão e identificar fatores associados à ocorrência deste agravo em uma unidade de internação de hospital privado de Minas Gerais. Metodologia: Estudo descritivo, transversal, de prevalência. Foi avaliada uma amostra de 169 pacientes internados no referido hospital. Resultados: Da amostra de 169 pacientes, nove desenvolveram lesão por pressão, sendo que um paciente desenvolveu três lesões, totalizando 11 lesões na casuística. A prevalência foi de 5,3\%. Três lesões foram classificadas como Estágio 1, sete como Estágio 2 e uma como Estágio 3. Conclusão: A taxa de prevalência observada estava abaixo da taxa dos trabalhos referenciados por este estudo. Esse dado pode estar relacionado a treinamento de manejo e prevenção de lesão por pressão, que foi ministrado aos profissionais de enfermagem do hospital em questão, durante o segundo semestre de 2017. Assim, a educação sobre o tema prevenção de lesão por pressão deve ser estimulada nas unidades de internação.

Descritores: Lesão por Pressão; Cuidados de Enfermagem; Prevalência.

\section{PREVALENCE OF PRESSURE INJURY IN INJURED PATIENTS IN A PRIVATE HOSPITAL OF THE STATE OF MINAS GERAIS}

Objectives: To estimate the prevalence of pressure injury and to identify factors associated with the occurrence of this injury in a private hospital in Minas Gerais. Methodology: descriptive, cross - sectional, prevalence study. A sample of 169 patients hospitalized at the hospital was evaluated. Results: From the 169 sample, nine patients developed pressure lesions, one patient developed three lesions, totaling 11 lesions in the sample. The prevalence was 5.3\%. Three lesions were classified as Stage 1 , seven as Stage 2 and one as Stage 3. Conclusion: The observed prevalence rate was below the rate of the studies referenced by this study. This data may be related to LPP management and prevention training, which was given to the nursing professionals of the hospital in question during the second half of 2017. Thus, in-service education is relevant for professionals and especially for patients.

Keywords: Pressure Ulcer, Nursing care, Prevalence.

\section{PREVALENCIA DE LESIÓN POR PRESIÓN EN PACIENTES INTERNADOS EN HOSPITAL PRIVADO DEL ESTADO DE MINAS GERAIS}

Objetivos: Estimar la prevalencia de lesión por presión e identificar factores asociados a la ocurrencia de este agravio en una unidad de internación de hospital privado de Minas Gerais. Metodología: Estudio descriptivo, transversal, de prevalencia. Se evaluó una muestra de 169 pacientes internados en el referido hospital. Resultados: De la muestra de 169, nueve pacientes desarrollaron lesión por presión, siendo que un paciente desarrolló tres lesiones, totalizando 11 lesiones en la casuística. La prevalencia fue del 5,3\%. Tres lesiones fueron clasificadas como Etapa 1, siete como Etapa 2 y una como Etapa 3. Conclusión: La tasa de prevalencia observada estaba por debajo de la tasa de los trabajos referenciados por este estudio. Este dato puede estar relacionado con entrenamiento de manejo y prevención de LPP, que fue impartido a los profesionales de enfermería del hospital en cuestión, durante el segundo semestre de 2017. Así, la educación en servicio se muestra relevante para los profesionales y principalmente para los profesionales pacientes asistidos.

Descriptores: Úlcera por Presión; Cuidados de enfermeria; Prevalencia. 


\section{INTRODUÇÃO}

As Lesões por Pressão (LP) consistem em lesões localizadas na pele e/ou no tecido subjacente, normalmente sobre uma proeminência óssea, ou relacionadas a dispositivos de cuidado à saúde, resultantes de pressão sustentada, incluindo a associação entre esta e cisalhamento ${ }^{(1)}$. O desenvolvimento das LP consiste em problema mundial em todos os níveis assistenciais de saúde, afetando pessoas de todos os grupos etários ${ }^{(2)}$.

O National Pressure Ulcer Advisory Panel (NPUAP)(3) anunciou a mudança na terminologia Úlcera por Pressão para Lesão por Pressão e a atualização da nomenclatura dos estágios do sistema de classificação. As variáveis referentes ao risco de LP consideram seis parâmetros da Escala de Braden: percepção sensorial, umidade, atividade, mobilidade, nutrição e fricção e/ou cisalhamento. Ainda segundo o NPUAP, as LP classificam-se em: Lesão por Pressão Estágio 1, Lesão por Pressão Estágio 2, Lesão por Pressão Estágio 3. Lesão por pressão Estágio 4, Lesão por Pressão Não Classificável, Lesão por Pressão Tissular Profunda, Lesão por Pressão Relacionada a Dispositivo Médico e Lesão por Pressão em Membranas Mucosas.

Nas unidades de internação hospitalares a realidade ainda é preocupante. Trabalho sobre aspectos clínicos e epidemiológicos da LP em pacientes hospitalizados mostrou que, no período de um mês, foram avaliados 197 pacientes que estavam internados sendo que 53 apresentaram LP, configurando uma prevalência pontual de $26,09 \%{ }^{(4)}$. Outro trabalho(5), visando mensurar a prevalência de LP em pacientes internados em três hospitais de ensino apresentou média anual da prevalência geral de 10,1\%.

No Brasil, o Ministério da Saúde instituiu o Programa Nacional de Segurança do Paciente por meio da Portaria no 529, de 1 으 de abril de 2013. Neste, um dos objetivos é a diminuição da ocorrência da LP(6)

A avaliação do risco para o desenvolvimento de LP pode ser realizada pela Escala de Braden, instrumento amplamente utilizado para esse fim $^{(7)}$. $O$ exame físico diário do paciente hospitalizado - incluindo a aplicação da Escala de Braden pode prevenir ou evitar agravamentos da LP quando esta já está instalada. No entanto, apesar de ser um tema de grande atenção no âmbito do cuidado de enfermagem, a incidência e prevalência mundial de LP permanece elevada, fato que comprova a necessidade de novas pesquisas com vistas a aperfeiçoar medidas preventivas e terapêuticas ${ }^{(8)}$

Um trabalho eficaz de prevenção pressupõe o conhecimento da etiologia e também da realidade da instituição. A prevenção de LP em pacientes hospitalizados tem sido apresentada como um dos indicadores de qualidade da assistência prestada pela equipe como um todo(9).

Os enfermeiros e principalmente os enfermeiros estomaterapeutas, por serem referências no cuidado a pacientes com lesões, incontinências, cateteres, drenos e estomias devem proporcionar um cuidado que envolva a prevenção e manejo das LP. Esta caracteriza um indicador negativo de qualidade do cuidado. É avaliada internacionalmente como evento adverso e representa importante desafio para o cuidado em saúde por contribuir com o aumento da morbidade, da mortalidade, tempo, custos do tratamento de saúde e afetar elevado número de pessoas $^{(10)}$

Como agente ativo no processo de cuidado ao paciente e suas necessidades, o enfermeiro deve se apropriar de conhecimento que forneçam embasamento para a prevenção de eventos adversos, dentre elas a LP. Problemas de saúde como as LP ainda são frequentes nas instituições hospitalares devido à assistência realizada de forma aleatória, sem existência de padronização dos cuidados, falta de atualização dos profissionais quanto ao uso de métodos para prevenção e falta de implantação de protocolos de ações que visem facilitar a assistência prestada ao paciente(1l)

O planejamento das ações de enfermagem auxilia na prevenção e tratamento das LP podendo assim, minimizar os custos financeiros, desgaste físico e emocional dos pacientes e familiares causados por este agravo(9).

Considerando-se a gravidade da ocorrência de LP em pacientes hospitalizados, observa-se que ainda são insuficientes os programas de prevenção deste agravo, bem como prevenção de complicações do mesmo, quando já instalado. Observa-se também, de maneira geral, que os fatores de risco para LP são pouco valorizados pela equipe de saúde. No hospital campo de estudo, há pacientes internados com um ou mais fatores de risco para LP. No entanto, há carência de dados numéricos que documentem a ocorrência de LP, o que dificulta a aplicação e criação de novos protocolos, no sentido de se evitar a ocorrência de LP, ou mesmo minimizar seus danos quando eventualmente ocorrem. A implementação de cuidados frente a este evento certamente sofre influências de dados e registros sobre pacientes internados.

Assim, este trabalho teve como objetivos estimar a prevalência de lesão por pressão e identificar fatores associados à ocorrência deste agravo em uma unidade de internação de hospital privado de Minas Gerais.

\section{METODOLOGIA}

\section{Tipo de estudo}

Estudo descritivo, transversal, de prevalência.

\section{Local do estudo}

Unidade de internação médico-cirúrgica de hospital privado e de ensino do Estado de Minas Gerais. 


\section{Participantes da pesquisa}

A fonte de dados foi constituída por prontuários eletrônicos de pacientes internados durante o primeiro semestre de 2018, em unidade hospitalar médico-cirúrgica e que apresentavam risco de desenvolvimento de LP. Os prontuários foram escolhidos aleatoriamente. O cálculo da amostra foi realizado considerando-se o número de 300 leitos da referida unidade.

\section{Critérios de inclusão e exclusão}

Realizou-se a escolha aleatória de 169 prontuários de pacientes que estiveram internados no primeiro semestre de 2018 e que fossem maiores de dezoito anos. Foram excluidos prontuários de pacientes menores de 18 anos.

\section{Coleta de dados}

A coleta de dados foi realizada durante o mês de junho de 2018, utilizando-se instrumento de coleta contendo as seguintes variáveis: demográficas: idade, sexo, situação laboral (aposentado, trabalhando fora) e cor da pele (branco, pardo, preto, outro);

epidemiológicas: tempo de internação, diagnóstico médico registrado no prontuário e ocorrência ou não de internação na unidade de terapia intensiva; relacionadas à LP: presença de LP e caracterização desta quanto à localização e ao estágio; classificação da LPP: foi utilizada a classificação de Lesão por Pressão Estágio 1, Lesão por Pressão Estágio 2, Lesão por Pressão Estágio 3, Lesão por pressão Estágio 4, Lesão por Pressão Não Classificável, Lesão por Pressão Tissular Profunda, Lesão por Pressão Relacionada a Dispositivo Médico e Lesão por Pressão em Membranas Mucosas (NPUAP) $)^{(3)}$

\section{Análise dos dados}

Os dados coletados foram registrados em uma planilha do aplicativo Microsoft Excel, versão Windows 98, por meio da técnica de dupla digitação. Posteriormente, os dados foram exportados e analisados no programa Statistical Package for Social Science (SPSS), versão 11.5 para análise estatística, sendo utilizado o cálculo da taxa de prevalência e das frequências absoluta e relativa para a descrição das variáveis coletadas.

\section{Aspectos éticos}

O projeto foi submetido à Câmara Departamental do Departamento de Enfermagem Básica (ENB) da Escola de Enfermagem da UFMG. Posteriormente, foi submetido ao Comitê de Ética em Pesquisas (COEP) do referido hospital, que o aprovou sob número CAAE 90070818130015125 (Plataforma Brasil). Como foram consultados os prontuários e não os pacientes, foi solicitado ao COEP desta instituição à dispensa do Termo de Consentimento Livre e Esclarecido.

\section{RESULTADOS}

Foi avaliada uma amostra de 169 pacientes internados no hospital cenário desta pesquisa. Destes 169, nove (09) pacientes desenvolveram LP. Observa-se uma prevalência de $5,3 \%$ (intervalo de $95 \%$ de confiança $=[2,5 \%$; 9,9\%]). Oito pacientes estavam acometidos com uma LP cada e um paciente foi identificado com três lesões, o que totalizou 11 lesões. Do total das 11 lesões, três lesões foram classificadas como Estágio 1, sete como Estágio 2 e uma em Estágio 3.

Quanto à localização das LP, 73\% destas estavam localizadas na região sacral, 9\% no calcâneo direito, 9\% no calcâneo esquerdo e $9 \%$ na região interglútea.

A idade variou de 35 anos (mínimo) há 103 anos (máximo), com uma média de 71 anos. Quanto ao sexo, cinco pacientes eram homens e quatro eram mulheres. Em relação à situação laboral, cinco estavam aposentados e quatro trabalhavam fora. No que tange à cor, cinco pacientes foram identificados como pardos e quatro como brancos. Não houve negros e indígenas na casuística.

Nos fatores associados à ocorrência de LP, há forte associação entre o tempo de internação do pacientes e lesões por pressão. Já a idade não se associou à LP. A Tabela 1 ilustra este dado:

Tabela 1 - Análise univariada para identificação de fatores associados às lesões por pressão em pacientes internados em hospital privado do Estado de Minas Gerais, Jan-Jun/2018:

\begin{tabular}{llcccc}
\hline & $\begin{array}{l}\text { Lesão por } \\
\text { pressão }\end{array}$ & n & Média & $\begin{array}{c}\text { Desvio } \\
\text { padrão }\end{array}$ & Valor-p \\
\hline Idade (anos) & Sim & 9 & 71 & 18,6 & 0,168 \\
& Não & 160 & 61 & 18,9 & \\
& & & & & \\
Tempo de internação & & 9 & 71 & 43,8 & $<0,001$ \\
(dias) & Sim & 160 & 15 & 24,3 & \\
& Não & & & & \\
\hline
\end{tabular}

Identificaram-se nos nove pacientes que desenvolveram LP os seguintes diagnósticos: três casos de neoplasias, uma doença renal em estágio final, um caso de dor em MMII a esclarecer, um caso de fadiga a esclarecer, um caso de dor pélvico-abdominal a esclarecer, um caso de aterosclerose das artérias de extremidades e um caso de dor em MMSS a esclarecer

Quanto à análise da possivel associação de variáveis categóricas com LP em pacientes internados no hospital em questão, somente o tempo de internação dos pacientes se associou às lesões por pressão. A Tabela 2 apresenta estes dados: 
Tabela 2 - Análise da possível associação de variáveis categóricas com LPP em pacientes internados em hospital privado do Estado de Minas Gerais, Jan-Jun/2018:

\begin{tabular}{|c|c|c|c|c|c|c|}
\hline Variável & categoria & $\begin{array}{c}\text { Total de } \\
\text { pacientes }\end{array}$ & $\begin{array}{c}\text { Número de } \\
\text { pacientes com } \\
\text { lesão por } \\
\text { pressão }\end{array}$ & Prevalência & $\begin{array}{c}\text { Prevalência } \\
\text { Relativa }\end{array}$ & Valor-p \\
\hline \multirow{2}{*}{$\begin{array}{l}\text { Idade }>75 \\
\text { anos? }\end{array}$} & Sim & 45 & 4 & $8,9 \%$ & \multirow[t]{2}{*}{2,2} & \multirow[t]{2}{*}{0,249} \\
\hline & Não & 124 & 5 & $4,0 \%$ & & \\
\hline \multirow{2}{*}{$\begin{array}{l}\text { Tempo de } \\
\text { internação } \\
>30 \text { dias? }\end{array}$} & Sim & 23 & 7 & $30,4 \%$ & \multirow[t]{2}{*}{22,2} & \multirow[t]{2}{*}{$<0,001$} \\
\hline & Não & 146 & 2 & $1,4 \%$ & & \\
\hline \multirow[t]{2}{*}{ Sexo } & Masculino & 77 & 5 & $6,5 \%$ & \multirow[t]{2}{*}{1,5} & \multirow[t]{2}{*}{0,733} \\
\hline & Feminino & 92 & 4 & $4,3 \%$ & & \\
\hline \multirow[t]{3}{*}{$\begin{array}{l}\text { Ocupação } \\
\text { principal }\end{array}$} & Do lar & 11 & 1 & $9,1 \%$ & \multirow[t]{2}{*}{1,8} & \multirow[t]{2}{*}{0,463} \\
\hline & ocupação & 158 & 8 & $5,1 \%$ & & \\
\hline & Belo & & & & \multirow{3}{*}{1,8} & \multirow{3}{*}{0,505} \\
\hline \multirow[t]{2}{*}{ Procedência } & Horizonte & 90 & 6 & $6,7 \%$ & & \\
\hline & Outras cidades & 79 & 3 & $3,8 \%$ & & \\
\hline
\end{tabular}

\section{DISCUSSÃO}

Dos 169 pacientes avaliados, 09 desenvolveram LP. Observou-se uma prevalência de 5,3\%, lembrando-se que a prevalência mede a proporção de pessoas que estão doentes no momento de se avaliar a doença na população, ou seja, não há tempo para acompanhamento(12). Estudo sobre prevalência de LP realizado com pacientes de hospital público no Brasil(10) apresentou prevalência de 40\%, dado muito acima do encontrado neste estudo. Também outro estudo, realizado em hospital filantrópico de Curitiba, com $\mathrm{N}$ de 87 pacientes apresentou prevalência pontual de LP de 40\%. Ressalta-se que em 2017, os profissionais de enfermagem do hospital cenário desta pesquisa receberam treinamento sobre prevenção e manejo de LP por profissional expert na área. Infere-se que os profissionais encontram-se instrumentalizados para prevenir e minimizar as LP de seus pacientes.

Oito pacientes apresentaram apenas uma LP, enquanto um paciente apresentou três LP. O paciente que desenvolveu três LP era homem, 61 anos, cor branca e suas LP encontravamse na região sacral, calcâneo direito e calcâneo esquerdo, permanecendo internado por 68 dias, sendo restrito ao leito por mais tempo. Em trabalho sobre custos de LP(1) os autores observaram que os pacientes encontravam-se restritos ao leito e as lesões por pressão localizavam-se na maioria em região sacral. Segundo os autores, a restrição ao leito apresentada por todos os pacientes pode ter colaborado para o surgimento de lesões por pressão, todas localizadas na região sacral. A restrição ao leito reduz a capacidade de alívio de pressão nas proeminências ósseas, mantendo os fatores de intensidade e duração da pressão.

A distribuição do local das lesões foi: sacral (73\%), interglútea (9\%), calcâneo direito (9\%) e calcâneo esquerdo (9\%). Em revisão integrativa(13), os autores encontraram artigos nos quais os locais mais comuns da LPP foram as regiões sacral e sacrococcígea, seguidas pelos calcâneos e interglúteos, corroborando os dados deste estudo. Também estudo sobre LP em pacientes acamados (14) detectou que as regiões anatômicas de maior ocorrência de LP foram sacro coccígeas, com $70 \%$ da casuística, seguida de maléolo e trocânter.

Três lesões foram classificada como Estágio 1, sete como estágio 2 e uma como estágio 3, totalizando 11 lesões, lembrando que um paciente apresentou três lesões. A maioria das LP foi classificada como estágio 2. A literatura é controversa, uma vez que estudo sobre LP(15) encontrou que $72 \%$ das úlceras se classificaram em estágio 1 .

A idade variou de 35 a 103 anos, com média de 71 anos. Revisão de literatura sobre $\mathrm{LP}^{(16)}$ pontua que pacientes idosos ou com lesão medular apresentam risco maior no desenvolvimento de LP e, consequentemente, constituem a população estatisticamente mais acometida, fato corroborado neste estudo.

Quanto à cor, quatro pacientes (45,5\%) apresentavam cor branca e cinco $(55,5 \%$.) apresentavam a cor parda. Não houve pessoas negras na casuística. A literatura considera que a pele negra tem maior resistência à agressão externa causada pela umidade e fricção, somada à dificuldade em identificar lesões de estádio 1 em indivíduos negros. Também não houve pacientes indígenas, o que pode ser justificado pela localização do hospital, uma capital brasileira ${ }^{(17)}$.

No que se refere aos fatores associados à ocorrência de LP, há forte associação entre o tempo de internação do pacientes e lesões por pressão, com o valor de $P<0,001$. Artigo de revisão integrativa de literatura apresenta o tempo de internação como um dos fatores associados à ocorrência de $L P^{(13)}$

Quanto à doença de base, houve três casos de neoplasias (33,3\%). Trabalho realizado sobre características e prevalência de pacientes em cuidados paliativos estudou 64 pacientes, todos com algum tipo de câncer. Destes, a prevalência de LPP foi de $18,8 \%{ }^{(18)}$. Segundo os autores, pacientes com doenças avançadas, incluindo aqueles em cuidados paliativos, costumam apresentar LP, feridas resultantes de lesão de pele ou tecido adjacente causada por pressão, cisalhamento e/ou fricção, que, no entanto, podem e devem ser evitadas. Neste trabalho, houve um paciente com doença renal em estágio final, também em cuidados paliativos.

No que e refere à análise da possível associação de variáveis categóricas com LP em pacientes internados no hospital cenário desta pesquisa, durante o período analisado, o tempo de internação do pacientes se associou às lesões por pressão $(P<0,001)$. Estudo aponta que, a partir do 15 의 dia de internação, todos os pacientes internados apresentam algum risco para o aparecimento de LP(19). O mesmo estudo destaca que o maior tempo médio de internação foi verificado na unidade no qual os pacientes apresentaram maior risco 
para UP, segundo Escala de Braden. Entretanto, não houve associação entre aparecimento de LP e tempo de internação.

\section{Limitações do estudo}

Observaram-se algumas limitações deste estudo, sendo a principal delas a ausência de alguns dados clínicos e sóciodemográficos de pacientes no hospital cenário deste estudo. Dados como escolaridade não foram preenchidos em todos os prontuários, o que impossibilitou a caracterização sócio demográfica completa dos pacientes. Também não foram preenchidos os dados referentes a peso e altura, o que impossibilitou o cálculo de IMC, limitando a caracterização clínica dos pacientes estudados. Lembra-se que os registros ou anotações de enfermagem consistem na forma de comunicação escrita de informações pertinentes ao cliente e aos seus cuidados. Entende-se que os registros são elementos imprescindiveis no processo de cuidado humano, visto que, quando redigidos de maneira que retratam a realidade a ser documentada, possibilitam à comunicação permanente, podendo destinar-se a diversos fins como para: pesquisas, auditorias, processos jurídicos, planejamento e outros ${ }^{(20)}$.

\section{Contribuições do estudo para a prática}

Este estudo contribuirá com dados e informações sobre a ocorrência de LP, subsidiando outras pesquisas e instrumentalizando profissionais da saúde para um melhor enfrentamento deste agravo.

\section{CONCLUSÃO}

De acordo com os objetivos, conclui-se que estes foram parcialmente alcançados, fato relacionado às limitações descritas no final da discussão deste trabalho.

Observou-se uma prevalência de 5,3\%, uma taxa abaixo da taxa de prevalência dos trabalhos sobre LP referenciados por este estudo. Esse dado, no nosso entender, está relacionado a treinamento de manejo e prevenção de LP, recebido pelos profissionais de enfermagem do hospital cenário deste estudo, no segundo semestre de 2017. Dessa forma, a educação em serviço mostra-se relevante, para os profissionais e principalmente para os pacientes, receptores do cuidado.
Sugere-se a melhora do processo de comunicação escrita nos prontuários da instituição, pois a falta de dados em prontuário foi o grande limitador deste trabalho. Sugerese também que novas pesquisas sejam realizadas em hospital de grande porte, uma vez que o tema LP é relevante, sendo seu estudo fundamental para a melhoria da qualidade da assistência.

\section{Contribuição dos autores}

Fernanda de Carvalho: Concepção, desenho, análise, interpretação dos dados e redação do artigo. Miguir Terezinha Vieccelli Donoso: Concepção, desenho, análise, interpretação dos dados, redação do artigo, revisão crítica e revisão final.Braulio Roberto Gonçalves Marinho Couto: Cálculo estatístico, análise, interpretação dos dados e redação do artigo.Selme Silqueira de Matos: Desenho, análise, interpretação dos dados, redação do artigo.Lilian Kelly Barbosa Lima: Concepção, desenho, análise, interpretação dos dados e redação do artigo.Érica Pertussati: Concepção, coleta de dados nos prontuários, análise e interpretação dos dados. 


\section{REFERÊNCIAS}

1. Silva DRA, Bezerra SMG, Costa JP, Luz MBA Lopes VCA Noqueira LT. : Curativos de lesões por pressão em pacientes criticos: análise de custos. Rev Esc Enferm USP. [Internet]. 2017 [citado 2019 Abr 3]:51:e03231.Disponivel em: http:// www.scielo.br/pdf/reeusp/v51/pt_1980-220X-reeusp-51-e03231.pdf

2. Otto C, Schumacher B, Wiese LPL, Ferro C, Rodrigues RA. Fatores de risco para o desenvolvimento de lesão por pressão em pacientes críticos. Enferm Foco. [Internet]. 2019 [citado 2019 Abr 3]: 10(1):07-11. Disponivel em: http://revista.cofen. gov.br/index.php/enfermagem/article/view/1323/485

3. National Pressure Ulcer Advisory Panel. Pressure Ulcer Stages Revised. Washington, 2016; Disponivel em:http://WWW.npuap.org/about-us/

4. Galvão NS, Lopes Neto D, Oliveira APP. Aspectos epidemiológicos e clínicos de pacientes com úlcera por pressão internados em uma instituição hospitalar. Estima. [Internet]. 2015 [citado 2019 Fev 18]; 13(3):sp. Disponivel em: https://www. revistaestima.com.br/index.php/estima/article/view/106

5. Melleiro MM, Tronchin DMR, Baptista CMC, Braga AT, Paulino A, Kurcgant $P$. Indicadores de prevalência de úlcera por pressão e incidência de queda de paciente em hospitais de ensino do municipio de São Paulo. Rev Esc Enferm USP. [Internet]. 2015 [citado 2019 Mar 01]; 49(Esp2):55-59. Disponivel em: http://www. scielo.br/pdf/reeusp/v49nspe2/1980-220X-reeusp-49-spe2-0055.pdf

6. Ministério da Saúde (BR). Resolução - RDC № 36, de 25 de julho de 2013. Institui ações para a segurança do paciente em serviços de saúde e dá outros providências. Brasilia (DF): Ministério da Saúde; 2013

7. Serpa LF, Santos VLCG. Campanili TCGF, Queiroz M. Predictive Validity of the Braden Scale for Pressure Ulcer Risk in Critical Care Patients. Rev Latino-Am Enfermagem [Internet]. 2011 Fev [cited 2019 Fev 28]; 19(1):50-7. Disponivel em: http://www.scielo.br/pdf/rlae/vl9nl/pt_08.pdf

8. Medeiros ABF, Lopes CHAF, Jorge MSB. Análise da prevenção e tratamento das úlceras por pressão propostos por enfermeiros. Rev Esc Enferm USP. [Internet] ulceras por pressāo propostos por enfermeiros. Rev Esc Enferm USP. [Internet]
2009 [citado 2019 Mar 01]; 43(1):223-28. Disponivel em: http://www.scielo.br/ 2009 [citado 2019 Mar 01]; 43(1):223-28. Disponivel em: http://ww
scielo.php?script=sci_arttextEpid=S0080-62342009000100029

9. Ascari RA, Veloso J, Silva OM, Kessler AMJ, Schwaab G. Úlcera por pressão: um desafio para a enfermagem. Brazilian Journal of Surgery and Clinical Research. [Internet]. 2014 [citado 2019 Abr 01]; 6(1):11-16. Disponivel em: https://www.mastereditora.com.br/periodico/20140301_132755.pdf

10. Bernardes RM, Caliri MHL. Prevalência de úlcera por pressão em hospital de emergência: estudo transversal. Revista Estima. [Internet]. 2016 [citado 2019 Mar 03]; 14(2):99. Disponivel em:

https://www.revistaestima.com.br/index.php/estima/article/view/383

11. Dantas ALM. Araújo JDB. Ferreira PC. Valença CN, Diniz KD, Carvalho ALB.

Prevenção de Úlceras por Pressão Segundo a Perspectiva do Enfermeiro Intensivista. Rev. Enferm UFPE on line. [Internet]. 2013 [citado 2019 Mar 23]; 7(1): 706-12. Disponivel em: http://www.repositorio.ufc.br/handle/riufc/7183
12. Fajardo-Gutierrez A. Medición en epidemiologia: prevalencia, incidencia. riesgo, medidas de impacto. Rev. alerg. Méx. [Internet]. 2017 [citado 2019 Abr 03]; 64(1):109-20. Disponivel em: http://www.scielo.org.mx/scielo.php?script=sci_arttext8pid=S2448-91902017000100109

13. Souza RG, Oliveira TL, Lima LR, Stival MM. Fatores associados à úlcera por pressão (UPP) em pacientes criticos: revisão integrativa da literatura. Universitas: Ciências da Saúde. [Internet]. 2016 [citado 2019 Fev 14]; 14(1):77-84. Disponivel em: https://www. publicacoesacademicas uniceub.br/cienciasaude/article/viewFile/3602/3072 14. Pessoa EFR, Rocha JGSC, Bezerra SMG. Prevalência de úlcera por pressão em
pacientes acamados, cadastrados na Estratégia de Saúde da Familia: um estudo pacientes acamados, cadastrados na Estratégia de Saúde da Familia: um estudo Fev 13]; 4(1):14-18. Disponivel em: https://revistainterdisciplinar.uninovafapi.edu. br/revistainterdisciplinar/v4nl/pesquisa/p2_v4nl.pdf

15. Borghardt AT, Prado TNP, Bicudo DSC, Bringuente MEO. Úlcera por pressão em pacientes criticos: incidência e fatores associados. Rev Bras Enferm. [Internet]. 2016 [citado $2019 \mathrm{Abr}$ 03]: 69(3):460-7. Disponivel em: http://dx.doi. org/10.1590/0034-7167.2016690307i.

16. Luz SR, Lopacinski AC, Fraga R, Urban R. Ùlceras de pressão. Geriatria \& Gerontologia. [Internet]. 2010 [citado 2019 Mar 07]; 4(1):36-43. Disponivel em:www. ggaging.com/export-pdf/296/v4nla06.pdf

17. Blanes L, Duarte IS, Calil JÁ, Ferreira LM. Avaliação clínica e epidemiológica das úlceras por pressão em pacientes internados no Hospital. Rev Assoc Med Bras [Internet] 2004 [citado 2019 Abr 03]: 50(2):182-7. Disponivel em: http:// www.scielo.br/pdf/ramb/v50n2/20781

18. Queiroz ACCM, Mota,DDCF, Bachion MM, Ferreira ACM. Úlceras por pressão em pacientes em cuidados paliativos domiciliares: prevalência e caracteristicas. Rev. Esc. Enferm. USP. [Internet]. 2014 [citado 2019 Mar 07]: 48(2):264-71. Disponivel em: http://www.scielo.br/pdf/reeusp/v48n2/pt_0080-6234-reeusp-48-02-264.pdf

19. Gomes FSL, Bastos MAR, Matozinhos FP, Temponi HR, Velazquez-Meléndez G. Avaliação de risco para úlcera por pressão em pacientes críticos. Rev. Esc. Enferm. USP. [Internet]. 2011 [citado 2019 Abr 03]; 45(2):313-18. Disponivel em:http:// www.scielo.br/scielo.php?script=sci_arttext\&pid=\$0080-62342011000200002

20. Matsuda LM et al.. Anotações/Registros de Enfermagem: instrumento de comunicação para a qualidade do cuidado? Revista de Eletrônica de Enfermagem. [Internet]. 2006 [citado 2019 Abr 03]; 8(3): 415-21. Disponivel em: http://www.fen. ufg.br/revista/revista8_3/v8n3al2.htm 